\title{
Many-To-One Stable Matching: Geometry and Fairness
}

\author{
Jay Sethuraman * Chung-Piaw Teo ${ }^{\dagger} \quad$ Liwen Qian ${ }^{\ddagger}$
}

March 2004; revised January 2005, January 2006

\begin{abstract}
Baïou and Balinski (2000) characterized the stable admissions polytope using a system of linear inequalities. The structure of feasible solutions to this system of inequalities - fractional stable matchings - is the focus of this paper. The main result associates a geometric structure with each fractional stable matching. This insight appears to be interesting in its own right, and can be viewed as a generalization of the lattice structure (for integral stable matchings) to fractional stable matchings. In addition to obtaining simple proofs of many known results, the geometric structure is used to prove the following two results: first, it is shown that assigning each agent their "median" choice among all stable partners results in a stable matching, which can be viewed as a "fair" compromise; second, sufficient conditions are identified under which stable matchings exist in a problem with externalities, in particular, in the stable matching problem with couples.
\end{abstract}

\section{Introduction}

The stable marriage problem and its variants have been studied extensively over the last few decades. Beginning with the pioneering work of Gale and Shapley [17], this problem has captured the attention of researchers and practitioners in several disciplines such as computer science, economics, mathematics, and operations research. The multidisciplinary nature of these problems has led to a thorough understanding of many aspects such as the design of efficient algorithms to find a stable matching, the structure of all solutions to a given stable matching instance, etc. In fact, by now there are several well-developed approaches to the stable matching problems: the combinatorial/algorithmic approach as summarized in the books of Knuth [25] and Gusfield \& Irving [18]; the linear programming approach initiated by Vande Vate [40], and further developed by Rothblum [37], Roth, Rothblum \& Vande Vate [35], Teo \& Sethuraman [39], Baïou \& Balinski [6] and Fleiner [16]; the fixed-point approach of Subramanian [38] and Feder [12,13]; an alternative fixed-point approach of Adachi [1]; yet

\footnotetext{
${ }^{*}$ IEOR Department, Columbia University, New York, NY. Email: jay@ieor.columbia.edu

${ }^{\dagger}$ Sungkyunkwan Graduate School of Business, Sungkyunkwan University, Seoul, Korea; and NUS Business School, National University of Singapore, Singapore 119260. Email: bizteocp@nus.edu.sg.

${ }^{\ddagger}$ Singapore-MIT Alliance Program, National University of Singapore, Singapore 119260. Email: scip9366@nus.edu.sg
} 
another fixed-point approach due to Fleiner [14]; and a graph-theoretic approach due to Balinski \& Ratier [7, 8] and Baïou \& Balinski [5].

In addition to its elegant theory, a particularly appealing feature of the stable matching model is its applicability. In fact, starting with the work of Roth [29], applications to the National Resident Matching Program (NRMP) and related labor markets have given rise to interesting questions, resulting in a better understanding of the theory. Moreover, insights from this theory have been useful in the redesign of the stable matching algorithm used by the NRMP [32]. An introduction to the theory of stable matchings with particular emphasis on applications to labor markets is elegantly summarized in Roth \& Sotomayor [33]; recent work in this direction include Roth \& Peranson [31, 32], Cantala [11], and Klaus \& Klijn [22].

This close interaction between applications and theory continues to this day, and is an inspiration for the questions we study, described next.

Problem description, Motivation, and Results. It has long been recognized that the (natural) stable matching mechanisms suggested by the "proposal" algorithm of Gale \& Shapley are biased, i.e., they compute the best stable matching for one side of the market, which incidentally is also the worst stable matching for the other side of the market. From its inception until 1997, the NRMP used the hospital-optimal matching mechanism as the basis of its allocation. However, increasing pressure from student bodies and others ${ }^{1}$ led to the redesign of the matching mechanism in 1998 [31, 32].

The inequitable treatment of the participants on different sides of the market persists in most known matching mechanisms. This has motivated the need for the design of fair stable matching mechanisms, which do not overtly favor one side of the market over the other. Considerable efforts have been devoted to finding "fair" stable matchings, including the egalitarian solution (minimize the sum of the ranks of the participants) and the minimum-regret solution (maximize the welfare of the participant worst-off in the matching) [18]. Nevertheless, these approaches are not satisfactory as they focus on socially optimal solutions, and ignore the issue of fairness at the individual level.

Motivated by "procedural" fairness considerations, Klaus and Klijn [22] analyze three probabilistic stable matching mechanisms: employment by lotto, proposed by Aldershof et al. [3]; the random order mechanism, proposed by Roth and Vande Vate [36] and Ma [26]; and the equitable random order mechanism, proposed by Romero-Medina [28]. Their analysis shows that the three mechanisms may give completely different outcomes. They also note that the associated probability distribution for each of these mechanisms need not be uniform on the set of stable matchings; in fact, not all stable matchings can arise from these random matching mechanisms. Klaus and Klijn [22] construct an example and showed that a stable matching that constitutes a perfect compromise between contrary preferences on both sides of the market may never result from random matching mechanisms. Curiously, the

\footnotetext{
${ }^{1}$ This took its clearest form in an exchange in the June 1995 issue of Academic Medicine, followed by position papers put out by the American Medical Students Association and others. Much of that discussion was oriented around results concerning "two-sided matching markets," of which the NRMP is a special case.
} 
perfect compromise solution identified there is the median solution (where the agents are assigned to their median stable partners), whose existence had in fact been established earlier in Teo and Sethuraman [39] for the one-to-one stable matching problem. The existence of such a solution is not obvious: consider the assignment obtained by pairing each agent with his or her median stable partner; that such an assignment should be a matching is itself surprising, so it is quite amazing that the resulting solution is not only a matching, but is also stable! In this paper, we establish the existence of such a median solution concept for the many-to-one model, thus proposing a new approach to address the asymmetry often observed in the stable matching problem. This result as well as the earlier result in [39] can be viewed as a generalization of an observation first made by Conway [18] on the lattice structure of stable matchings ${ }^{2}$

Several generalizations and extensions of the stable matching problem have been studied in the past because of potential applications to NRMP and other labor markets. Of particular interest is the one in which certain pairs of students ("couples") would like to be assigned to universities that are geographically close together. We identify natural conditions on the preferences under which the couples problem can be handled effectively. Note that in this case, a stable matching solution may not even exist. Furthermore, Klaus et al. [24] show that in the presence of couples, the current NRMP algorithm may fail to converge to a stable matching!

The common theme underlying our results is a refined understanding of the structure of "fractional" stable matchings. Baïou and Balinski [6] characterize the convex hull of all stable admissions solutions using linear inequalities in the natural assignment variables. We use the Baïou-Balinski formulation to show that the fractional stable admission solutions can be decomposed into convex combination of (integral) stable admission solutions in a simple way. As a by-product, our approach gives a simple visual proof of the integrality of the Baïou-Balinski formulation.

The main results in this paper are derived using a "bin-packing" theorem, obtained by packing the fractional solutions in suitable chosen order. Interestingly, this may be viewed as a continuous analogue of the bin-packing theorem used by Fleiner [14] to describe the polytope of the more general many-to-many stable matchings. However, the approach used by Fleiner depends on a clever fixed point argument, but the underlying inequalities are obtained through an iterative algorithm. The description of the polytope is thus implicit. The Baïou-Balinski formulation is explicit and is thus a

\footnotetext{
${ }^{2}$ Since the first version of this manuscript, Fleiner [15] and Klaus \& Klijn [23] (independently of each other) have provided (similar) proofs of the existence of the median stable matching, using the lattice structure. We deduce the existence of a median solution by working with fractional solutions, whereas they work directly with the set of (integral) many-to-one stable matchings. Their proof can be viewed as a "discrete" analog of ours. An anonymous referee observed the following simple proof of existence: construct the natural expanded one-to-one instance obtained by making $q_{u}$ copies of university $u$, each copy of $u$ having the same preferences as $u$ itself; To break ties, we allow the students to rank the copies in an arbitrary but fixed manner. The stable matchings for the expanded one-to-one instance can be shown to be shown to be in one-one correspondence with the stable matchings for the many-to-one problem. This allows us to use the results derived in [39], on top of Theorem 7, to derive a simple proof for the existence of the median stable matching solution.
} 
more convenient and natural approach for our goal.

Organization of the paper. We introduce the stable admissions polytope in section 2. In section 3 , we provide a geometric structure to the fractional stable admissions solutions. Section 4 uses this structure to design a fair stable matching mechanism to the many-to-one stable admission problem. We also describe how the stable matching problem with couples can be addressed using this approach. We end with a brief summary.

\section{The stable admissions model}

An instance of the stable admissions problem consists of two sets of agents, the set $U=\left\{u_{1}, u_{2}\right.$, $\left.\ldots, u_{|U|}\right\}$ of "universities" and the set $A=\left\{a_{1}, a_{2}, \ldots, a_{|A|}\right\}$ of "students." Each agent has a strict, transitive, preference ordering of the acceptable agents on the other side of the market, i.e., those agents on the other side of the market that it prefers to remaining unmatched. We assume without loss of generality that (i) $u$ finds $a$ acceptable if and only if $a$ finds $u$ acceptable, and in this case, we say that $(u, a)$ is an acceptable pair; and (ii) university $u$ finds at least $q_{u}$ students acceptable, and each student finds at least one university acceptable. Let $\Gamma \subseteq U \times A$ denote the set of acceptable pairs. Finally, associated with university $u$ is a positive integer $q_{u}$ representing its quota, the interpretation being university $u$ is allowed to admit up to $q_{u}$ students. Note that we consider the somewhat restrictive model of responsive preferences in which the universities have preferences over individual students, not over groups of students. We refer the reader to Roth \& Sotomayor [33, Chapter 6] for interesting discussions on this issue ${ }^{3}$.

A matching $\mu$ for a stable admissions problem is a subset of $\Gamma$ such that each university $u$ appears in at most $q_{u}$ pairs and each student $a$ appears at most once in $\mu$. For convenience, we let $\mu(a)$ represent the university that student $a$ is assigned to, and $\mu(u)$ represent the set of students assigned to university $u$. We let $\mu(a)=\{a\}$ if student $a$ is unmatched in $\mu$.

A matching $\mu$ is stable if there is no incentive for any pair $(u, a)$ to deviate from $\mu$. That is, there is no pair $(u, a) \in \Gamma$ such that student (i) student $a$ prefers university $u$ to $\mu(a)$ and (ii) university $u$ prefers to add student $a$ to its set of students, possibly at the expense of another (less-preferred) student. (If $a$ is unmatched in $\mu$, then (i) is trivially satisfied.)

By the classical result of Gale and Shapley [17], every instance of the stable admissions problem admits a stable matching. The stable admissions problem with $q_{u}=1$ for all $u \in U$ is called the stable marriage problem.

\footnotetext{
${ }^{3}$ It is conceivable that the results in this paper remain valid for more general and realistic classes of preferences over groups of students (cf. Abdulkadiroğlu [4]). The proof techniques needed for the extension, however, will be entirely different, as our argument relies on a characterization of the convex hull of the set of stable solutions.
} 


\subsection{Definitions and Notation}

The symbols $>_{u}$ and $>_{a}$ will indicate the preference orderings of university $u$ and student $a$ respectively; in particular, $a>_{u} b$ iff university $u$ prefers student $a$ to student $b$, and $a \geq_{u} b$ iff $a>_{u} b$ or $a=b$. The shaft, $S(u, a)$, is defined for every $(u, a) \in \Gamma$ as

$$
S(u, a)=\left\{(u, b) \in \Gamma: b \geq_{u} a\right\} .
$$

The tooth, $T(u, a)$, is defined for every $(u, a) \in \Gamma$ as

$$
T(u, a)=\left\{(v, a) \in \Gamma: v \geq_{a} u\right\}
$$

For any set of $q_{u}$ students $\left\{a, a_{1}, \ldots, a_{q_{u}-1}\right\}$ with $a_{i}>_{u} a$ for all $i$, the $\operatorname{comb} C\left(u, a, a_{1}, \ldots, a_{q_{u}-1}\right)$ is the union of the shaft $S(u, a)$, the tooth $T(u, a)$, and the teeth $T\left(u, a_{i}\right), i=1, \ldots, q_{u}-1$. Such a comb is said to be based at $(u, a)$. Let $C_{u}$ be the collection of all combs based at $(u, a)$, for some $a \in A$, and let $\mathcal{C}=\cup_{u \in U} C_{u}$. To any stable admissions solution $\mu$, we can associate a $|\Gamma|$-vector $x_{\mu}$ (or $x$, if the stable matching $\mu$ is clear from the context) by letting $x_{\mu}(u, a)=1$, if $(u, a) \in \mu$, and $x_{\mu}(u, a)=0$

otherwise. For any $\hat{\Gamma} \subseteq \Gamma$, we let $x(\hat{\Gamma})=\sum_{(u, a) \in \hat{\Gamma}} x(u, a)$. A pair $(u, a) \in \Gamma$ blocks $\mu$ if (i) $u$ prefers $a$ to at least one of its assigned students in $\mu$, or if $u$ is assigned fewer than $q_{u}$ students; and (ii) $a$ prefers $u$ to its assigned university in $\mu$, or if $a$ is unmatched. We can express this condition using the notions of shaft and tooth defined earlier:

$$
(u, a) \text { blocks } \mu \text { iff } x_{\mu}(S(u, a)) \leq q_{u}-1 \text { and } x_{\mu}(T(u, a))=0 .
$$

The convex hull of the incidence vectors of all stable admissions solutions is the stable admissions polytope (of that instance), discussed next.

\subsection{The stable admissions polytope}

Let $C\left(u, a, a_{1}, \ldots, a_{q_{u}-1}\right)$ be a comb in $C_{u}$. Every stable admissions solution $x$ must satisfy the comb inequality

$$
x\left(C\left(u, a, a_{1}, \ldots, a_{q_{u}-1}\right)\right) \equiv x(S(u, a))+x(T(u, a) \backslash\{(u, a)\})+\sum_{i=1}^{q_{u}-1} x\left(T\left(u, a_{i}\right) \backslash\left\{\left(u, a_{i}\right)\right\}\right) \geq q_{u},
$$

first described by Baïou and Balinski [6]. Otherwise, $x(S(u, a))<q_{u}$, and $x\left(T\left(u, a^{\prime}\right)\right)=0$ for some $a^{\prime} \in\left\{a, a_{1}, a_{2}, \ldots, a_{q_{u}-1}\right\}$. As $a^{\prime} \geq_{u} a, x\left(S\left(u, a^{\prime}\right)\right) \leq x(S(u, a)) \leq q_{u}-1$; By $(1)$, the pair $\left(u, a^{\prime}\right)$ is a blocking pair in $x$. It is a simple matter to verify that for any unstable matching $\mu$, one can find a violated comb inequality: let $(u, a)$ be a blocking pair, and $\hat{a}$ be the student who is ranked $q_{u}$ th in $u$ 's preference list. If $u$ prefers $\hat{a}$ to $a$, any comb based on $(u, a)$ that includes the teeth $T\left(u, a^{\prime}\right)$ 
for all $\left(u, a^{\prime}\right) \in \mu, a^{\prime}>_{u} a$ is violated; otherwise, the (only) comb based on $(u, \hat{a})$ is violated. These considerations show that the integer programming problem (see [6])

$$
\begin{aligned}
& \sum_{u:(u, a) \in \Gamma} x_{u, a} \leq 1, \quad \forall a \in A, \\
& \sum_{a::(u, a) \in \Gamma} x_{u, a} \leq q_{u}, \quad \forall u \in U, \\
& x(C)=\sum_{(u, a) \in C} x_{u, a} \geq q_{u}, \quad \forall C \in C_{u}, u \in U . \\
& x_{u, a} \in\{0,1\}, \quad \forall(u, a) \in \Gamma
\end{aligned}
$$

is an exact formulation of the stable admissions problem. Let $P_{F S A}$ denote the set of all solutions to the associated (fractional) linear programming relaxation in which the constraints $x_{u, a} \in\{0,1\}$ are replaced by $x_{u, a} \geq 0$. Let $P_{S A}$ be the convex hull of all stable matchings to the stable admissions problem. Clearly, $P_{S A} \subseteq P_{F S A}$. The main result of Baïou and Balinski [6] is that, in fact, $P_{S A}=P_{F S A}$.

In the next section we study the structure of fractional solutions of the stable admissions polytope; we exploit this structure in the following sections to derive a host of interesting results, including a simple proof that $P_{S A}=P_{F S A}$.

\section{Geometry of fractional stable admissions}

\subsection{Decomposition}

To motivate our main result, it is useful to think of each university $u$ as owning $q_{u}$ "bins," each of size (= height) 1; each bin represents a seat and is indexed by the pair $(i, u)$. Each $x_{u a}>0$ will be treated as an "item" to be packed into one of the bins owned by university $u$. Bins are filled in the usual way (bottom-to-top); all items are packed in decreasing preference order of the university, following the procedure described next.

In phase 0, each university $u$ packts its (at most) $q_{u}$ best items, (at most) one in each of its bins. (If $u$ is fractionally matched with fewer than $q_{u}$ students, then some of its bins will remain unfilled.) Bins that are completely empty at the end of this phase are excluded from the rest of the packing process. Phase $t$ (for $t=1,2, \ldots$ ) of the procedure consists of (a) identifying the set, $L_{t}$, of bins with the maximum available space; and (b) assigning one item to each of the bins in $L_{t}$. The assignment of the items to the bins within a phase proceeds in a sequence of steps, indexed by $l=1,2, \ldots,\left|L_{t}\right|$. By our assumption, if bin $(i, u) \in L_{t}$ is considered in step $l$, university $u$ 's best remaining item is packed into it. We have not specified the order in which the bins are examined within each phase. As it turns out, the order does not matter.

At the end of any step of the packing procedure, let $M_{i, u}$ be the set of students assigned to bin $i$ of university $u$; and let $a_{i, u}$ be university $u$ 's least preferred student in $M_{i, u}$. In terms of our bin-packing 
analogy, $a_{i, u}$ is the student occupying the top-most position in bin $(i, u)$. Our main result is the following.

Theorem 1 Consider any $x \in P_{F S A}$. Let $M_{i, u}$ be the set of students assigned to bin $i$ of university $u$ at the end of any step of the packing procedure. Let $a_{i, u}$ be university u's least preferred student in $M_{i, u}$. Then,

(a) For all $(i, u)$ with $M_{i, u} \neq \emptyset$,

$$
x\left(M_{i, u} \backslash a_{i, u}\right)+x\left(T\left(u, a_{i, u}\right)\right)=x\left(M_{i, u}\right)+x\left(T\left(u, a_{i, u}\right) \backslash a_{i, u}\right)=1
$$

(b) If item $(v, a)$ is packed but $(u, a)$ is not, then a prefers $u$ to $v$.

(c) At the end of any phase, the $a_{i, u}$ are all distinct. In particular, for each $a \in A$ with $\sum_{v \in U} x_{v a}>0$, there is some $(i, u)$ such that $a \equiv a_{i, u}$.

Theorem 1(a) plays a similar role to the stability constraints in the one-to-one stable marriage problem. Interestingly, the packing procedure decomposes the $q_{u}$ positions for university $u$ implicitly into $q_{u}$ copies, each of quota 1 , and identifies the set of students used to fill the $i$ th position in university $u$. This essentially reduces the stable admission problem to a one-to-one stable-marriage like problem. Note that the universities pack the students into the bins in decreasing preference order, but Theorem 1(b) states that the packing process assigns universities to students in increasing preference order. Theorem 1(c) states that at the end of each phase, the items at the top of each bin forms a valid assignment.

Before proving the theorem, it will be useful to consider an illustrative example.

\subsection{Example}

Consider the following example, adapted from Section 1.6.5 of Gusfield and Irving [18]. There are 5 universities and 11 students, with the preference lists and the quotas given in Tables 1 and 2. There are a total of 7 stable matchings for this particular problem instance, and these are listed in Table 3.

Let $x_{M_{i}}$ be the (integral) stable matching corresponding to $M_{i}$ in the stable admission polytope, and let $x=\left(\sum_{i=1}^{7} x_{M_{i}}\right) / 7$ be a fractional solution in the stable matching polytope. We illustrate the packing procedure for this solution $x$.

Phase 0. Initially all the bins are empty, so $L_{1}$ consists of all the bins; each of these bins will have an item in it at the end of the first phase. Recall that items are packed by the universities in decreasing preference order.

Consider university $u_{1}$, which owns four bins. Its four best students in $x$ are $a_{9}, a_{11}, a_{5}$, and $a_{4}$, with the corresponding "values" being $3 / 7,1 / 7,1$, and $2 / 7$ respectively. Thus, the four bins owned 


\begin{tabular}{llllll}
\hline$a_{1}$ & $u_{3}$ & $u_{1}$ & $u_{5}$ & $u_{4}$ & \\
$a_{2}$ & $u_{1}$ & $u_{3}$ & $u_{4}$ & $u_{2}$ & $u_{5}$ \\
$a_{3}$ & $u_{4}$ & $u_{5}$ & $u_{3}$ & $u_{1}$ & $u_{2}$ \\
$a_{4}$ & $u_{3}$ & $u_{4}$ & $u_{1}$ & $u_{5}$ & \\
$a_{5}$ & $u_{1}$ & $u_{4}$ & $u_{2}$ & & \\
$a_{6}$ & $u_{4}$ & $u_{3}$ & $u_{2}$ & $u_{1}$ & $u_{5}$ \\
$a_{7}$ & $u_{2}$ & $u_{5}$ & $u_{1}$ & $u_{3}$ & \\
$a_{8}$ & $u_{1}$ & $u_{3}$ & $u_{2}$ & $u_{5}$ & $u_{4}$ \\
$a_{9}$ & $u_{4}$ & $u_{1}$ & $u_{5}$ & & \\
$a_{10}$ & $u_{3}$ & $u_{1}$ & $u_{5}$ & $u_{2}$ & $u_{4}$ \\
$a_{11}$ & $u_{5}$ & $u_{4}$ & $u_{1}$ & $u_{3}$ & $u_{2}$ \\
\hline
\end{tabular}

Table 1: Preference lists for the students

\begin{tabular}{|c|c|c|c|c|c|c|c|c|c|c|c|c|}
\hline \multicolumn{13}{|c|}{ capacity } \\
\hline$(4)$ & $u_{1}$ & $a_{3}$ & $a_{7}$ & $a_{9}$ & $a_{11}$ & $a_{5}$ & $a_{4}$ & $a_{10}$ & $a_{8}$ & $a_{6}$ & $a_{1}$ & $a_{2}$ \\
\hline (1) & $u_{2}$ & $a_{5}$ & $a_{7}$ & $a_{10}$ & $a_{6}$ & $a_{8}$ & $a_{2}$ & $a_{3}$ & $a_{11}$ & & & \\
\hline (3) & $u_{3}$ & $a_{11}$ & $a_{6}$ & $a_{8}$ & $a_{3}$ & $a_{2}$ & $a_{4}$ & $a_{7}$ & $a_{1}$ & $a_{10}$ & & \\
\hline (2) & $u_{4}$ & $a_{10}$ & $a_{1}$ & $a_{2}$ & $a_{11}$ & $a_{4}$ & $a_{9}$ & $a_{5}$ & $a_{3}$ & $a_{6}$ & $a_{8}$ & \\
\hline (1) & $u_{5}$ & $a_{2}$ & $a_{4}$ & $a_{10}$ & $a_{7}$ & $a_{6}$ & $a_{1}$ & $a_{8}$ & $a_{3}$ & $a_{11}$ & $a_{9}$ & \\
\hline
\end{tabular}

Table 2: Quotas and preference lists for the universities

\begin{tabular}{llllllllllll}
\hline Matching & $a_{1}$ & $a_{2}$ & $a_{3}$ & $a_{4}$ & $a_{5}$ & $a_{6}$ & $a_{7}$ & $a_{8}$ & $a_{9}$ & $a_{10}$ & $a_{11}$ \\
\hline$M_{1}$ & $u_{3}$ & $u_{1}$ & $u_{4}$ & $u_{3}$ & $u_{1}$ & $u_{3}$ & $u_{2}$ & $u_{1}$ & $u_{4}$ & $u_{1}$ & $u_{5}$ \\
$M_{2}$ & $u_{1}$ & $u_{3}$ & $u_{4}$ & $u_{3}$ & $u_{1}$ & $u_{3}$ & $u_{2}$ & $u_{1}$ & $u_{4}$ & $u_{1}$ & $u_{5}$ \\
$M_{3}$ & $u_{3}$ & $u_{1}$ & $u_{5}$ & $u_{3}$ & $u_{1}$ & $u_{3}$ & $u_{2}$ & $u_{1}$ & $u_{4}$ & $u_{1}$ & $u_{4}$ \\
$M_{4}$ & $u_{1}$ & $u_{3}$ & $u_{5}$ & $u_{3}$ & $u_{1}$ & $u_{3}$ & $u_{2}$ & $u_{1}$ & $u_{4}$ & $u_{1}$ & $u_{4}$ \\
$M_{5}$ & $u_{5}$ & $u_{3}$ & $u_{3}$ & $u_{4}$ & $u_{1}$ & $u_{3}$ & $u_{2}$ & $u_{1}$ & $u_{1}$ & $u_{1}$ & $u_{4}$ \\
$M_{6}$ & $u_{5}$ & $u_{4}$ & $u_{3}$ & $u_{1}$ & $u_{1}$ & $u_{3}$ & $u_{2}$ & $u_{3}$ & $u_{1}$ & $u_{1}$ & $u_{4}$ \\
$M_{7}$ & $u_{4}$ & $u_{4}$ & $u_{3}$ & $u_{1}$ & $u_{1}$ & $u_{3}$ & $u_{2}$ & $u_{3}$ & $u_{1}$ & $u_{5}$ & $u_{1}$ \\
\hline
\end{tabular}

Table 3: List of all Stable Matchings 

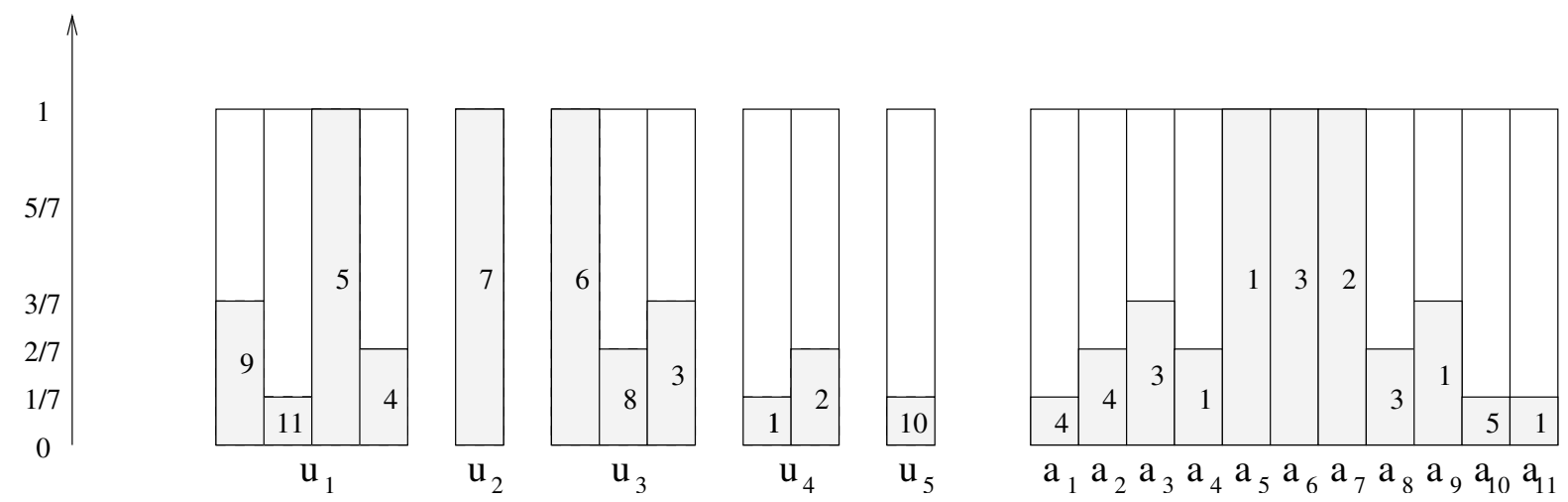

Figure 1: Bins at the end of Phase 0

by $u_{1}$ contain these four items as shown in Figure 1 . For convenience, we may also assume that each student also owns a bin in which items are packed: whenever item $(u, a)$ is packed into bin $(i, u)$, we may also assume that item $(u, a)$ is packed into the bin owned by student $a$. Thus, students $a_{9}, a_{11}$, $a_{5}$, and $a_{4}$ each pack an item into their bins. In terms of this picture, it is easy to interpret the three statements of Theorem 1: part (b) simply says that this packing, when viewed from the point of view of the students, proceeds in increasing preference order; given this, part (a) says that the occupied space in any bin with student $a$ on top plus the available space in student $a$ 's bin is exactly 1 ; and part (c) says that at the end of any phase, the students on top of the bins owned by the universities are all distinct. All of these properties are easily verified from Figure 1, which shows the state of the bins at the end of phase 0 .

Phase 1. The set $L_{2}$ will consist of all the bins that have the maximum available space; from Figure 1 , it is clear that $L_{2}$ consists of bin 2 of $u_{1}$, bin 1 of $u_{4}$ and the bin owned by $u_{5}$. During phase 1 , each of these three bins will have an item assigned to it. In fact, the top students in these bins will rearrange themselves, so that part (c) continues to hold. University $u_{1}$ will pack its most preferred remaining items, which happens to be student $a_{10}$ (value 6/7); similarly, universities $u_{4}$ and $u_{5}$ will pack respectively items involving students $a_{11}$ (value $4 / 7$ ) and $a_{1}$ (value $2 / 7$ ) respectively. The state of the bins at the end of phase 1 is shown in Figure 2; it is easy to verify that parts (a)-(c) of Theorem 1 continue to hold.

End of packing process. By repeating the above packing procedure, the final state of the bins (after Phase 4) is shown in Figure 3. 


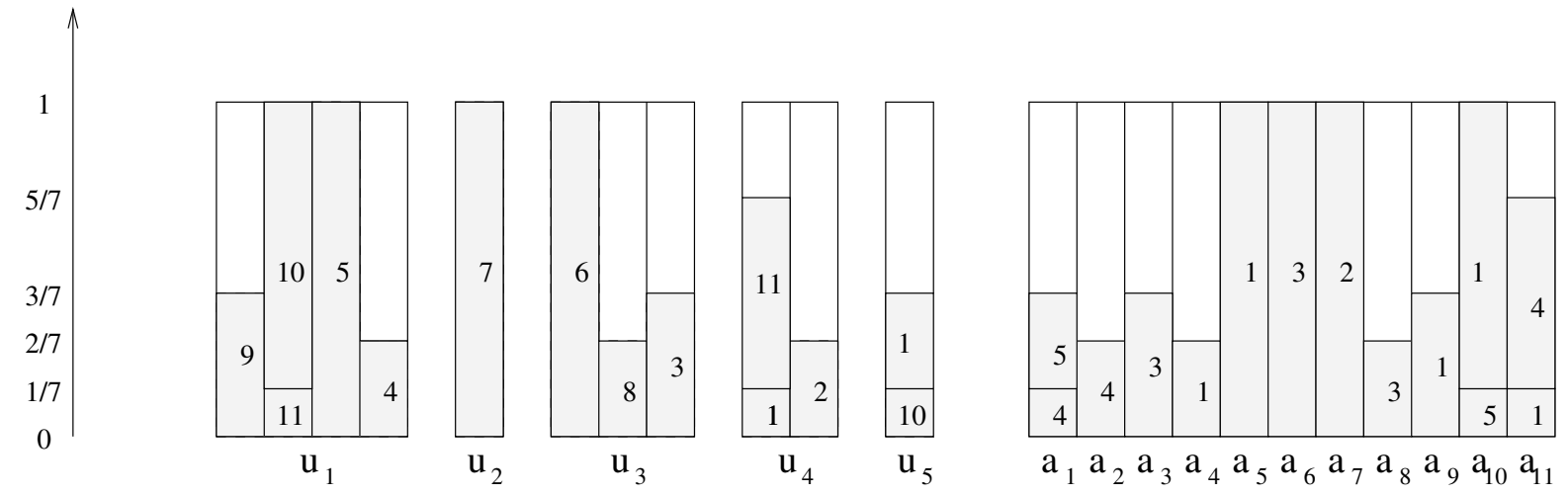

Figure 2: Bins at the end of Phase 1
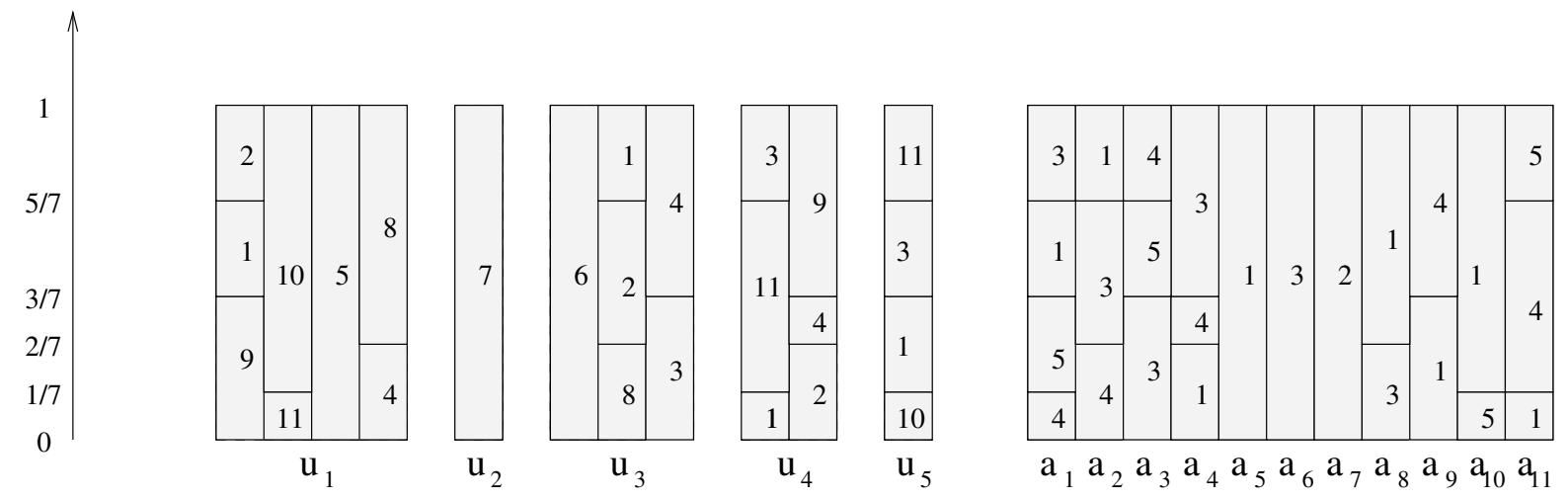

Figure 3: Bins at the end of packing procedure 


\subsection{Proof of Theorem 1}

Let $x \in P_{F S A}$. We say that a particular university-student pair $(u, a)$ is matched (under $x$ ) if $x_{u a}>0$; we say that they are fractionally matched, if $0<x_{u a}<1$.

It will be useful to understand the conditions under which a particular university-student pair is fractionally matched.

Lemma 2 Suppose $(k, l)$ is fractionally matched under $x$, i.e. $x_{k l} \in(0,1)$. Then,

$$
\sum_{j \in A} x_{k j}=q_{k}, \text { and } \sum_{i \in U} x_{i l}=1
$$

Proof. The necessary ingredients to prove this already appear in Baïou and Balinski [6]. Let $B_{u}(x)$ be $u$ 's $q_{u}$ most preferred students among the students it is matched to under $x$; if $u$ is matched with fewer than $q_{u}$ students, we let $B_{u}(x)$ be the set of all students that $u$ is matched to. Let $A_{u}(x)$ be the set of all students for whom $u$ is the most preferred university they are matched to under $x$.

Two simple facts will be useful in the proof:

$$
x(T(u, a))=1, \quad \forall a \in B_{u}(x),
$$

and

$$
x(T(u, a))=x_{u a}, \quad \forall a \in A_{u}(x) .
$$

The latter is immediate by definition. To see the former, consider the comb $C$ involving $u$ and the set of students $B_{u}(x)$, supplemented, if necessary, with additional students not matched to $u$ to get $q_{u}$ "teeth." Let $a_{1}, a_{2}, \ldots, a_{q_{u}}$ be these $q_{u}$ students. By definition,

$$
x(C)=\sum_{i=1}^{q_{u}} x\left(T\left(u, a_{i}\right)\right) .
$$

The LHS of Eq. (9) is at least $q_{u}$ by the comb inequality; whereas the RHS of Eq. (9) is at most $q_{u}$ by aggregating the appropriate student assignment constraints. Therefore,

$$
x\left(T\left(u, a_{i}\right)\right)=1 .
$$

In particular, $x(T(u, a))=1$ for each $a \in B_{u}(x)$.

We prove next that $\left|A_{u}(x)\right|=\left|B_{u}(x)\right|$ for all $u \in U$ : Clearly $B_{u}(x) \cap B_{v}(x)=\emptyset$ for $u \neq v$, otherwise there will be some $a \in B_{u}(x) \cap B_{v}(x)$ with $x(T(u, a))=1$ and $x(T(v, a))=1$. Hence

$$
\sum_{u \in U}\left|A_{u}(x)\right| \geq \sum_{u \in U}\left|B_{u}(x)\right|
$$

Therefore, to prove $\left|A_{u}(x)\right|=\left|B_{u}(x)\right|$, it is enough to show that $\left|A_{u}(x)\right| \leq\left|B_{u}(x)\right|$ for all $u \in U$. 
If $\left|B_{u}(x)\right|<q_{u}$, then $B_{u}(x)$ contains all the students who are matched to $u$. It is thus clear that $\left|A_{u}(x)\right| \leq\left|B_{u}(x)\right|$. Let $\left|B_{u}(x)\right|=q_{u}$, and suppose $\left|A_{u}(x)\right|>\left|B_{u}(x)\right|$. Consider the comb $C$ with the teeth chosen from $u$ 's $q_{u}$ best students from $A_{u}(x)$. By Eq. (8), all the positive $x$ components of comb $C$ involve university $u$; moreover, since some member of $A_{u}(x)$ is excluded from comb $C, x(C)<q_{u}$ by the university assignment constraint, which violates the comb-inequality for comb $C$. Therefore $\left|A_{u}(x)\right| \leq\left|B_{u}(x)\right|$.

Based on the preceding discussion, $\left|A_{u}(x)\right|=\left|B_{u}(x)\right|$ for each $u \in U$. The Lemma now follows from the following reasoning:

(a) If $\left|B_{u}(x)\right|<q_{u}$, then $\left|A_{u}(x)\right|=\left|B_{u}(x)\right|$ and Eqs. (7) and (8) imply $x_{u a}=1$ for all $a \in B_{u}(x)$. So any $u$ with $\left|B_{u}(x)\right|<q_{u}$ cannot have any fractionally matched students under $x$.

(b) Any fractionally-matched student $a$ belongs to some $A_{u}(x)$. Since the $A_{u}(x)$ are all disjoint and the $B_{v}(x)$ are all disjoint, and since $\sum_{u}\left|A_{u}(x)\right|=\sum_{v}\left|B_{v}(x)\right|$, a must also appear in $B_{v}(x)$ for some $v \in V$. By Eq. (7), $x(T(v, a))=1$; in particular, $\sum_{i \in U} x_{i a}=1$.

(c) If $x_{u a} \in(0,1)$, then $\left|B_{u}(x)\right|=\left|A_{u}(x)\right|=q_{u}$. The comb-inequality constraint on the comb $C\left(u, A_{u}(x)\right)$ and the university assignment constraint for $u$ show that $u$ must meet its quota exactly.

Lemma 2 ensures that any university $u$ with unfilled bins is only integrally matched to its students; In our packing procedure, such universities and the students they are matched to pack their items only at phase 0 , after which they play no role in the packing process. The residual instance now consists of all the universities that fill their quota, and the students they are matched with.

In the rest of this section, we can thus assume that every agent is "completely" matched: that is, $\sum_{u \in U} x_{u a}=1$ for each $a \in A$, and $\sum_{a \in A} x_{u a}=q_{u}$ for each $u \in U$.

Proof of Main Theorem: Our proof proceeds by induction on $t$. First, consider the case $t=0$. Since we start with a set of empty bins, all of the bins will be in $L_{0}$; at the end of the phase, it is clear that university $u$ will have (fractionally) packed its $q_{u}$ most preferred items, one in each of its bins. Let $B_{u}(x)$ be university $u$ 's $q_{u}$ most preferred students it is matched to under $x$.

Part (a) of the theorem now follows from observing that $M_{i, u}=a_{i, u}$. Part (b) is true because $x\left(T\left(u, a_{i, u}\right)\right)=1$, and $x_{u, a_{i, u}}>0$ for all $(i, u)$, and preferences are strict. Moreover, if $a_{i, u}=a_{j, v}=a$, then either $x(T(u, a))<1$ or $x(T(v, a))<1$, depending on whether $v>_{a} u$ or $u>_{a} v$; so, the $a_{i, u}$ are all distinct at the end of phase 0 , establishing the first statement of part (c). Since the number of students is the same as the total number of bins, the second statement of part (c) follows.

Suppose the theorem is true up to the end of phase $t$. We now establish the theorem for phase $t+1$. Let $\alpha$ be the maximum space available in any bin during phase $t+1$. Consider any item $(u, a)$ 
that has not yet been packed. From part (c), $a \equiv a_{j, v}$ for some bin $(j, v)$; from parts (a) and (b), we have

$$
x(T(u, a)) \leq x(T(v, a))-x_{v, a}=1-x\left(M_{j, v}\right) \leq \alpha,
$$

where the last inequality follows because each bin has at most $\alpha$ available space. Moreover,

$$
x(T(u, a))=\alpha \quad \text { implies } \quad(j, v) \in L_{t+1} .
$$

Suppose $(i, u) \in L_{t+1}$ is chosen in any step of phase $t+1$, and item $x_{u a}$ is to be packed into bin $(i, u)$ next. Consider the comb

$$
C_{u, a} \equiv C\left(u, a, a_{1, u}, a_{2, u}, \ldots, a_{i-1, u}, a_{i+1, u}, \ldots, a_{q_{u}, u}\right)
$$

which contains

- the teeth $T(u, a), T\left(u, a_{j, u}\right)$ for $j=1,2, \ldots, i-1, i+1, \ldots q_{u}$; and

- the shaft $S(u, a)=\{(u, a)\} \cup M_{1, u} \cup M_{2, u} \cup \ldots \cup M_{q_{u}, u}$.

By the comb inequality, we have

$$
q_{u} \leq x\left(C_{u, a}\right)=\sum_{j: j \neq i} x\left(T\left(u, a_{j, u}\right)\right)+x(T(u, a))+x\left(M_{i, u}\right)+\sum_{j: j \neq i} x\left(M_{j, u} \backslash\left\{a_{j, u}\right\}\right) .
$$

By induction, for $j \neq i$,

$$
x\left(T\left(u, a_{j, u}\right)\right)+x\left(M_{j, u} \backslash\left\{a_{j, u}\right\}\right)=1,
$$

which reduces the comb inequality to

$$
x(T(u, a))+x\left(M_{i, u}\right) \geq 1 .
$$

Since $(i, u) \in L_{t+1}$ and has not been packed earlier, $x\left(M_{i, u}\right)=1-\alpha$; from Eq. (11), $x(T(u, a)) \leq \alpha$. So the (reduced) comb inequality (13) must be satisfied as an equality. We next pack $(u, a)$ in bin $(i, u)$, and update $M_{i, u}$ and $a_{i, u}$, and observe that the reduced comb equality can be written as

$$
x\left(T\left(u, a_{i, u}\right)\right)+x\left(M_{i, u} \backslash a_{i, u}\right)=1,
$$

establishing part (a). Part (b) follows since if item $(u, a)$ is not packed but item $(v, a)$ is packed, then from Eq. (11),

$$
x(T(u, a)) \leq \alpha \leq x(T(v, a)) .
$$

Hence $a$ prefers $u$ to $v$.

Observe that $x(T(u, a))=\alpha$ for every item $(u, a)$ packed in phase $t+1$, and these are the least preferred items in their respective bins; this implies the least preferred items in the newly packed bins are all distinct. From (12), if a bin $(i, u)$ was not selected in phase $t+1$, its least preferred student could not have been part of any item packed in phase $t+1$. So the $\left\{a_{i, u}\right\}$ remain distinct at the end of phase $t+1$. 


\subsection{Consequences}

We begin this section by noting that the "rural hospitals" theorem is an easy consequence of Lemma 2.

Theorem 3 The set of universities that fill their position is the same in all stable matchings, as is the set of students assigned to some university; moreover, any university that does not fill its quota is assigned the same set of students in every stable matching.

Proof. Let $x$ be the average of all the stable matchings to the given stable admissions problem. Clearly, $x \in P_{F S A}$. Let $u$ be a university that does not fill its quota in some stable matching. Then, $\sum_{a \in A} x_{u a}<q_{u}$. By case (c) in the proof of Lemma 2, $\left|B_{u}(x)\right|<q_{u}$, for otherwise, $u$ must meet its quota exactly. Applying Lemma 2, part (a) to $x$, we see that $x_{u a}=1$ for all $a \in B_{u}(x)$, so $u$ must be matched to the same set of students in all stable matchings. Case (b) of Lemma 2 shows that the set of students assigned to a university is the same in all stable matchings.

Let $x \in P_{F S A}$. Because our goal is to highlight the structure of fractional solutions in $P_{F S A}$, we ignore universities that do not fill their quotas. As the proof of Lemma 2 and Theorem 3 show, such universities are assigned to the same set of students in every $x \in P_{F S A}$, and so can be safely ignored.

For any $\alpha \in[0,1)$, let

$$
I_{u}^{\alpha}(x)=\left\{a: 1-x(T(u, a)) \leq \alpha<1-x(T(u, a))+x_{u a}\right\}
$$

and $I^{\alpha}(x)=\cup_{u \in U} I_{u}^{\alpha}(x)$. By part (a) of Theorem 1, $I_{u}^{\alpha}(x)$ consists of those students whose assignment to their "bin" in university $u$ changes the occupied space in that bin from at most $\alpha$ to some quantity strictly greater than $\alpha^{4}$. Since each university fills all its bins, the following corollary of Theorem 1 is immediate.

Corollary 1 For any $x \in P_{F S A}$ and any $\alpha \in[0,1),\left|I_{u}^{\alpha}(x)\right|=q_{u}$.

Remark. Baïou and Balinski [6] observed that $I_{u}^{0}(x)=I_{u}^{1^{-}}(x)=q_{u}$ for any stable admissions solution $x$; Corollary 1 generalizes these observations.

Roth, Rothblum and Vande Vate [35] proved the following result for the stable marriage polytope using a primal-dual argument.

Lemma 4 Let $q_{u}=1$ for all $u \in U$. For any $x \in P_{F S A}, x_{u, a}>0$ implies

$$
x_{u, a}+x(S(u, a) \backslash(u, a))+x(T(u, a) \backslash(u, a))=1 .
$$

This result was crucial in proving that $P_{F S A}$ is exactly the convex hull of all stable marriage solutions [35, 39]. The following result generalizes Lemma 4 to the stable admissions setting; its proof is implicit in the proof of Theorem 1 and is therefore omitted.

\footnotetext{
${ }^{4}$ Geometrically, if we layout the items using our bin packing approach, to obtain a figure resembling Figure 3, then $I^{\alpha}(x)$ consists of those items in the layout which intersect with a horizontal line at $y=\alpha$.
} 
Lemma 5 For any $x \in P_{F S A}$, and any $\alpha \in[0,1)$, let $C_{\alpha, u}$ denote the comb obtained by using the $q_{u}$ students in $I_{u}^{\alpha}(x)$ as the bases for the teeth in the comb. Then, $x\left(C_{\alpha, u}\right)=q_{u}$.

We next show how the main result of Baïou and Balinski [6] follows as an immediate consequence of Theorem 1.

Theorem 6 The inequalities (3)-(6) determine the stable admissions polytope $P_{S A}$.

Proof. Given any $x \in P_{F S A}$, generate a number $\alpha \in(0,1]$ uniformly at random; for each university $u$, assign the $q_{u}$ students in $I_{u}^{\alpha}(x)$ to university $u$ to get an assignment $\mu_{\alpha}$. By part (c) of Theorem 1 , no student is assigned to two different universities under $\mu_{\alpha}$, so that $\mu_{\alpha}$ is a matching. Let $v=\mu_{\alpha}(b)$. We show next that $\mu_{\alpha}$ is stable.

Consider any student $b$ whom $u$ strictly prefers to at least one of its assigned students (say $a$ ) under $\mu_{\alpha}$. It is possible that $x(u, b)=0$. Consider the set $S^{*}=\left\{c: x(u, c)>0, c<_{u} b\right\}$, and let $b^{\prime}$ be the most preferred student in $S^{*}$, according to $u$. Since $a \in S^{*}$, we have $a \leq_{u} b^{\prime}$.

The item $\left(u, b^{\prime}\right)$ would have been packed not later than item $(u, a)$ by the packing procedure; moreover, at the beginning of the phase, say $t$, in which $\left(u, b^{\prime}\right)$ was packed, the space available in the bin occupied by student $b^{\prime}$ at university $u$, say $1-\beta$, must not be smaller than $1-\alpha$, otherwise $u$ cannot prefer $b^{\prime}$ to $a$.

If item $\left(u, b^{\prime}\right)$ is packed in phase $t>0$, then by Theorem 1 , we can always pick a comb $C$ based at $\left(u, b^{\prime}\right)$ which is tight: $x(C)=q_{u}$. $C$ can be obtained by choosing the least preferred student in each bin, at the moment when item $\left(u, b^{\prime}\right)$ is packed. We can form a new comb $C^{\prime}$ by shifting the base from $\left(u, b^{\prime}\right)$ to $(u, b)$. Note that $x(S(u, b))=x\left(S\left(u, b^{\prime}\right)\right)-x\left(u, b^{\prime}\right)$. Using the comb inequality $x\left(C^{\prime}\right) \geq q_{u}$, and the fact that $x(C)=q_{u}$, we have

$$
x(T(u, b)) \geq x\left(T\left(u, b^{\prime}\right)\right)=1-\beta \geq 1-\alpha .
$$

Hence $v \geq_{b} u$.

If item $\left(u, b^{\prime}\right)$ is packed in phase $t=0$, then $b^{\prime} \in B_{u}(x)$, i.e., $\left(u, b^{\prime}\right)$ is one of the $q_{u}$ most preferred items according to $u$. Note that the comb $C$ formed by the students in $B_{u}(x)$ is tight: $x(C)=q_{u}$. We can replace the most preferred student in $B_{u}(x)$ by $b$, to obtain a new comb $C^{\prime}$, with $x\left(C^{\prime}\right) \geq q_{u}$. Since $x\left(C^{\prime}\right)$ is simply the sum of the length of the $q_{u}$ teeth, we have $x(T(u, b)) \geq 1$. Hence again $v \geq_{b} u$.

The matching $\mu_{\alpha}$ obtained is thus stable. By construction, the expected value of $\mu_{\alpha}(u, a)$ is $x_{u, a}$. Hence any $x \in P_{F S A}$ can be written as $E\left(\mu_{\alpha}\right)$, i.e., convex combination of stable matching solutions. Integrality of the polytope is now immediate [39].

\section{Applications}

In the rest of this section, we use the geometry of fractional solutions of the stable admissions problem to address two natural applications: the design of a fair stable assignment mechanism, and the problem of assigning couples to "universities." 


\subsection{Fairness}

It has long been recognized in the economics literature that there are subtle differences between "procedurally-fair" and "outcome-fair" solution concepts (cf. Bolton et al. [9]). A procedurally-fair mechanism is useful in instances when the fairness of the process is viewed as more important than the fairness of the outcome. People feel affirmed if the adopted procedures treat them with respect and dignity, making it easier to accept outcomes, even the ones they do not like. However, in a matching market like the NRMP, it is not clear whether a procedurally-fair mechanism will be acceptable to all participants.

On the other hand, studies in which subjects divide resources between themselves and others have shown that most people preferred equality to either advantageous or disadvantageous inequality, see, for instance, Camerer and Thaler [10]. An important debate in this literature concerns which outcomefair solution concept is most appropriate for a particular situation, or whether any fair solution concept exists at all. The existence of a median stable matching in the one-to-one case, suggests that outcomefair resolution of the stable marriage problem may be possible. We will not attempt to formally justify the median stable marriage solution as an outcome-fair solution, but we note that in many experimental tests of bargaining solution concepts in the ultimatum game, Guth et al. [19]) show that "equal splitting" is the dominant behavior observed amongst the participants. Interestingly, the median solution proposed in this section shares many characteristics with the equal-split option in the bargaining problem - with a proper geometric layout of all the stable marriage solutions along a line, the median solution is obtained by splitting at the middle, with universities (men) and students (women) at opposite ends. A formal description now follows.

Suppose there are exactly $N$ stable matchings in an instance of the stable admissions problem, and let $x_{1}, \ldots, x_{N}$ be the stable solutions. For convenience, we assume $N$ is odd. Is there a way to choose a solution which treats all the participants in a fair manner? The starting point for the results described in this subsection is the following result due to Roth and Sotomayor [34] (see also Gusfield and Irving [18, Theorem 1.6.4, pp. 46] or Roth and Sotomayor [33, Theorem 5.27, pp. 161]). We include a simple proof here based on Theorem 1.

Theorem 7 Given 2 stable matchings $\mu$ and $\mu^{\prime}$, suppose university $u$ is assigned non-identical sets of students in $\mu$ and $\mu^{\prime}$. If $u$ prefers its least preferred student in $\mu \backslash \mu^{\prime}$ to its least preferred student in $\mu^{\prime} \backslash \mu$, then, $u$ prefers all the students in $\mu$ to any student in $\mu^{\prime} \backslash \mu$.

Proof. Let $x_{\mu}$ and $x_{\mu^{\prime}}$ denote the (integral) stable admission solutions corresponding to the matchings $\mu$ and $\mu^{\prime}$ respectively. Let $x=\left(x_{\mu}+x_{\mu^{\prime}}\right) / 2$, and consider the packing procedure on the fractional solution $x$. Note that if student $a$ is assigned to $u$ in $\mu \cap \mu^{\prime}$, then $x_{u a}=1$. Hence $(u, a)$ must be among the $q_{u}$ most preferred items packed at the initial phase, otherwise condition (a) of Theorem 1 will be violated. 
Let $s$ and $s^{\prime}$ be university $u$ 's least preferred students in $\mu \backslash \mu^{\prime}$ and $\mu^{\prime} \backslash \mu$ respectively. By assumption, $u$ prefers $s$ to $s^{\prime}$. Suppose $u$ prefers a student in $\mu^{\prime} \backslash \mu$ to some student in $\mu$. Then, $s$ cannot be one of $u$ 's $q_{u}$ most preferred students. From condition (c) of Theorem 1, the student $s$ must be among the $q_{u^{\prime}}$ most preferred students of some university $u^{\prime}$ in the matching $\mu^{\prime}$. Since $x\left(T\left(u^{\prime}, s\right)\right)=1, s$ prefers $u$ to $u^{\prime}$. Now, since $u$ prefers $s$ to $s^{\prime},(u, s)$ forms a blocking pair for the stable assignment $\mu^{\prime}$. This is a contradiction.

The key conclusion emerging from Theorem 7 is that any university either prefers all of its $\mu$ partners to any of its $\mu^{\prime}$-partners who are not also $\mu$-partners, or prefers all of its $\mu^{\prime}$-partners to any of its $\mu$-partners who are not also $\mu^{\prime}$-partners. In the former case, we say that university $u$ prefers $\mu$ to $\mu^{\prime}$, and in the latter, that it prefers $\mu^{\prime}$ to $\mu$. In particular, the worst student for university $u$ in its different stable assignments should all be different. Thus, each university can rank order all of its stable assignments in decreasing order of its worst student.

Consider the set of student assignments university $u$ receives under all stable solutions $x_{1}, \ldots, x_{N}$. By Theorem 7, $u$ can rearrange these solutions such that its worst student in each of the solutions appears in decreasing preference order, that is $x_{i}$ appears before $x_{j}$ if $u$ prefers its worst student in $x_{i}$ to its worst student in $x_{j}$. This induces a natural linear order on the set of solutions assigned to each university. We can thus define the median choice of each university: it is simply the median solution in the linear ordering of all stable solutions by the university ${ }^{5}$. Similarly, each student could rank order $x_{1}, x_{2}, \ldots, x_{N}$ in decreasing preference order based on the university she is assigned to. In this case, her median choice will be the median university in this linear ordering. A natural question then is: if each university and student is given their median choice, do we get a stable matching? In fact, it is not even obvious that assigning the median choice to each agent gives a valid assignment: there is no reason why the same student should not be the median choice of two different universities, or why two students should not have the same university as their median choice. Remarkably, the median choices of each agent results in a stable matching!

In the rest of this section, we assume that the given instance satisfies the conditions that all universities filled their quota, and all students are matched to a university. This is without loss of generality: universities that do not fill their quotas have only one set of students in all stable matchings, so such universities and the students they are matched to are not particularly interesting in studying fairness issues in stable matchings. Let

$$
x_{f}(u, a)=\frac{\sum_{j=1}^{N} x_{j}(u, a)}{N}
$$

where $x_{1}, \ldots, x_{N}$ are the set of all stable matchings to the given instance of the stable admissions problem.

\footnotetext{
${ }^{5}$ If $N$ is even, the median choice is not unique, but consists of two candidate solutions. Fortunately, the argument in this section can be easily modified to handle this case.
} 
Theorem 8 Let $M_{i, u}^{f}$ be the set of students assigned to bin $i$ of university $u$ by the packing procedure applied to the fractional stable matching $x_{f}$. Then, each stable matching contains exactly one student from each $M_{i, u}^{f}$.

Proof. The result is obviously true for all universities that do not fill their quota: such universities are matched to the same set of students in all stable matchings, and hence in $x_{f}$ as well. Let $u$ be a university that admits $q_{u}$ students in some (and hence all) stable matching. Clearly, $\sum_{a: a \in A} x_{f}(u, a)=$ $q_{u}$. Let $\mu_{1}>_{u} \mu_{2}>_{u} \ldots>_{u} \mu_{K}$ be the distinct sets of students that are assigned to university $u$ in some stable matching. Note that $\left|\mu_{i}\right|=q_{u}$ for all $i$.

We examine how the packing procedure packs $u$ 's bins starting with the fractional solution $x_{f}$. As before, the proof is by induction on the number of phases, the only change being we focus only on those phases that pack at least one of $u$ 's bins. (Our numbering of the phases also reflects this: phase $i$ is the $i$ th time university $u$ 's bins are packed by the packing procedure.) By Theorem 7, all the items associated with $\mu_{1}$ are packed in phase 1 , and each such item occupies a distinct bin. So the result is true at the end of phase 1 . Which items are packed during phase 2? Clearly, $u$ 's most preferred items that have not yet been packed are those in $\mu_{2} \backslash \mu_{1}$, and these will be packed in phase 2 . It is also clear that the bins into which these items are packed each contain one item of $\mu_{1} \backslash \mu_{2}$ : the remaining bins must contain items that are in both $\mu_{1}$ and $\mu_{2}$, so their values in $x_{f}$ will be strictly larger than the values of the items in $\mu_{1} \backslash \mu_{2}$. Thus, at the end of phase 2 , the items of $\mu_{2} \backslash \mu_{1}$ will each occupy a bin that now contain the items in $\mu_{1} \backslash \mu_{2}$; note that the stable assignments $\mu_{1}$ and $\mu_{2}$ contain exactly one item from each bin.

In phase 3 , the items in $S=\mu_{3} \backslash\left(\mu_{1} \cup \mu_{2}\right)$ will be packed. We claim that, in fact, $S=\mu_{3} \backslash \mu_{2}$. For otherwise, there must be a student $a$ such that $a \in \mu_{1}, a \in \mu_{3}$, but $a \notin \mu_{2}$. Since $u$ prefers $\mu_{1}$ to $\mu_{2}, u$ must prefer $a$ to any student in $a^{\prime} \in \mu_{2} \backslash \mu_{1}$; and since $u$ prefers $\mu_{2}$ to $\mu_{3}, u$ must prefer $a^{\prime}$ to any student in $\mu_{3} \backslash \mu_{2}$. In particular, $u$ must prefer $a^{\prime}$ to $a$, which is a contradiction. The bins with maximum available space are clearly those containing the items in $T=\mu_{2} \backslash \mu_{3}$. Because $S$ and $T$ have the same cardinality, and because each element of $T$ already appears in a distinct bin, each item in $S$ is assigned, by the packing procedure, to a bin containing an item of $T$. Now, each of $\mu_{1}, \mu_{2}$ and $\mu_{3}$ contains exactly one item from each bin. (This is because only the items in $S$ are newly assigned, none of which can be present in $\mu_{1} \cup \mu_{2}$.)

We are now ready to do an inductive proof: at phase $i$, let $S=\mu_{i} \backslash \mu_{i-1}, T=\mu_{i-1} \backslash \mu_{i}$; clearly,

$$
S=\mu_{i} \backslash\left\{\mu_{1} \cup \mu_{2} \cup \ldots \cup \mu_{i-1}\right\}
$$

Also, $S$ and $T$ are non-empty, have the same cardinality; all items in $T$ are in distinct bins, so the items in $S$ are assigned to distinct bins by the packing procedure, each containing a distinct item in $T$. Moreover, we have now ensured that the stable assignments $\mu_{1}, \mu_{2}, \ldots \mu_{i}$ all contain exactly one item from each bin; this is because only the items in $S$ are newly assigned, none of which can be present in any of the assignments $\mu_{1}, \mu_{2}, \ldots, \mu_{i-1}$. 
Remarks: Theorem 8 has also been derived independently by Fleiner [16] using somewhat similar methods. In the proof of Theorem 1, we showed how all the fractional partners of any university $u$ in a solution $x \in P_{F S A}$ can be partitioned into $q_{u}$ sets. Theorem 8 shows something stronger: that one can in fact construct a single partition of the students that works for all (fractional) solutions. Moreover, we can construct an associated stable marriage instance whose stable solutions will be in a one-to-one correspondence with the set of all stable admissions solutions of the original instance. The advantage of such a construction is clear: all of the results on the stable admissions problem can thus be reduced to that of the stable marriage problem by appealing to this transformation. Indeed, given this decomposition, it is a simple matter to find a compact linear formulation to express the convex hull of all stable matchings. The disadvantage is that constructing such a decomposition entails knowing the entire set of stable assignments for each university $u$.

The geometric structure of $x_{f}$ is extremely useful, as we can now immediately read off many possible stable admission solutions from the layout.

- University-optimal solution: Match $u$ to the $q_{u}$ students in $I_{0^{+}}(u)$. The solution obtained is university-optimal (best $q_{u}$ possible students are assigned) but each student is assigned her worst possible stable university. In the example in Figure 3, this corresponds to choosing all items touching the bottom of every bins in the figure.

- Student-optimal solution: Match $u$ to the $q_{u}$ students in $I_{1}(u)$. The students are assigned to their best possible stable university (i.e., student-optimal), and the universities are assigned to their worst possible set of students under any stable solution. In the example in Figure 3, this corresponds to choosing all items touching the top of every bin in the figure.

- Median solution: If $N$ is odd, we can define the median stable admission solution as follows: Match $u$ to the $q_{u}$ students in $I_{0.5}(u)$. The students are thus matched to their median stable university (counting multiplicity). By the packing procedure of section 3.1 and Theorem 8 , it is easy to see that the assignment corresponding to $I_{0.5}(u)$ is exactly the assignment where each university is matched with its median set of students. If $N$ is even, using the packing decomposition it is straightforward to get a similar result for markets with an even number of players: just set $\alpha=0.5+\epsilon$ or $\alpha=0.5-\epsilon$, and each agent will be assigned to one of two median sets of partners. In the example in Figure 3, this corresponds to choosing all items crossed by the line $\alpha=0.5$, i.e., occupying the halfway mark of every bin. The matching obtained: $u_{1} \leftarrow$ $\{1,5,8,10\}, u_{2} \leftarrow\{7\} ; u_{3} \leftarrow\{2,4,6\} ; u_{4} \leftarrow\{9,11\} ; u_{5} \leftarrow\{3\}$. This corresponds to the solution $M_{4}$, which is stable. $M_{4}$ is thus the median stable solution.

This discussion can be summarized into the following result:

Theorem 9 There exists a stable solution to the many-to-one stable matching problem with the following properties: 
- Assign each student to the median university, among all universities assigned to in the stable solutions;

- Assign each university its median set of students, among all stable admission solutions, and where the set of students assigned are ranked ordered based on the ranking of the worst assigned student in each solution.

Proof: Let $\mu_{1}>_{u} \mu_{2}>_{u} \ldots>_{u} \mu_{K}$ be the distinct sets of students that are assigned to university $u$ in some stable matching. Note that WLOG, we may assume that $\left|\mu_{i}\right|=q_{u}$ for all $i$. Let $k_{i}$ be the

number of times $\mu_{i}$ appears in all the stable solution. Hence $\sum_{i=1}^{K} k_{i}=N$. Suppose $N$ is odd. The median solution is $\mu_{k^{*}}$ where

$$
k^{*}=\operatorname{argmin}\left\{j: \sum_{i=1}^{j} k_{i} \geq \frac{N+1}{2}, \sum_{i=1}^{j-1} k_{i} \leq \frac{N-1}{2}\right\} .
$$

As in the proof to Theorem 7, we examine the packing procedure by focusing on the phases where bins associated with $u$ are being packed. We have proven in Theorem 7 that in phase $i$, items in $\mu_{i} \backslash \mu_{i-1}$ are being packed into the bins. In the beginning of phase $k^{*}$, the maximum available space in the bins are greater than 0.5 , but the addition of the items in $\mu_{k^{*}} \backslash \mu_{k^{*}-1}$ decreases the maximum available space on all bins to less than 0.5. Hence the set of students assigned to $u$, under $I_{0.5}(u)$, are exactly the students corresponding to the items in $\mu_{k^{*}} \backslash \mu_{k^{*}-1}$, and $\mu_{k^{*}} \cap \mu_{k^{*}-1}$. Hence the $q_{u}$ students in $I_{0.5}(u)$ are exactly the students in $\mu_{k^{*}}$.

The existence of a matching mechanism that assigns all participants their median choice is indeed surprising. One of the limitations of this mechanism though is that all the stable matching solutions must be enumerated before a median solution can be constructed. This can theoretically be obtained by enumerating all the corner points of the polytope given by the LP characterization of the stable admission polytope or by combinatorial methods. Finding an efficient method to compute the median solution remains a challenging open problem.

\subsection{Admissions with couples}

Finally, we consider the stable admissions problem with couples. This is an extension of the stable admission problem to incorporate externalities, where the preferences of the students may depend on the matching produced. In particular, where the stable admission problem includes pairs of couples, whose preferences (ranking of the universities) depends also on where their spouses are assigned to under the matching.

Consider for instance the example discussed in 3.2. Suppose further that $a_{4}$ and $a_{10}$ are a "couple." Although both prefer university $u_{3}$ to the rest of the hospitals, their preferences may change if one of them is not assigned to $u_{3}$. In particular, if university $u_{3}$ is very far from $u_{1}$, whereas $u_{4}$ and $u_{5}$ are nearby, then $a_{4}$ and $a_{10}$, who prefer to stay together, may rank the assignment $\left\{\left(u_{5}, a_{4}\right),\left(u_{4}, a_{10}\right)\right\}$ 
above the assignment $\left\{\left(u_{3}, a_{4}\right),\left(u_{1}, a_{10}\right)\right\}$, although individually they preferred the assigned universities in the latter assignment.

To handle the externalities introduced by the preferences of the couples, one common approach is to allow the couples to submit joint rankings of the pairs of universities they would like to be assigned to (cf. [33] and the references therein). We can define an analogous notion of stability in this problem. Unfortunately, many of the appealing results for the stable marriage problem do not carry over to the case where couples express preferences over pairs of positions. For example [2]:

(i) There are instances of the couples problem that do not have a stable matching;

(ii) Even if an instance of the couples problem has a stable matching, it may not have a university optimal or student optimal stable matching;

(iii) In an instance of the couples problem in which the preference lists are not complete, there may be stable matchings which leave different numbers of positions unfilled.

Klaus and Klijn [21] shows recently that stable solutions exist for the couple problem whenever the couples have "weakly responsive" preferences 6 . However, the definition of "weakly responsive" preferences essentially ensures that the couple preferences can be captured using suitably defined individual preferences, thus reducing the problem to the classical stable admissions problem without couples. This guarantees the existence of stable matching. Unfortunately, these assumptions on the preference profiles, which guarantee existence of a stable solution, may violate the most important consideration - that couples wish to be assigned to universities in close geographical proximity. Klaus and Klijn [21] further show that any slight deviation from the weakly responsiveness assumption easily results in instances where the stable admission problem will have no feasible solution. Interestingly, their example basically allow a couple to reverse their ranking, taking into account the geographical considerations. This suggests that any preference profiles submitted by couples that take into account the geographical considerations are likely to lead to instances where stable matching does not exist.

Cantala [11] also considers a class of realistic preferences in which (i) the "universities" are partitioned into regions; (ii) each couple first decides on a ranking of the regions (on which each couple agrees), and then ranks the universities within each region; their individual rankings of the universities within a given region may be different; and (iii) their preferences are otherwise independent, and each of them only cares about the university they are assigned to. Even under such severe restrictions on the class of preferences, Cantala [11] shows that a stable matching may not exist.

The literature so far seems to indicate that the stable admissions problem with couples may not have feasible solutions if realistic preferences of the couples (based on geographical proximity of the joint assignment) are to be modeled. In the rest of this section, we propose a different approach to handle this problem: Instead of allowing the couples to submit joint ranking of the universities, we

\footnotetext{
${ }^{6}$ cf. B. Klaus and F. Klijn (2005): Corrigendum: Stable Matchings and Preferences of Couples, for a minor correction
} 
accept only individual ranking of the universities, and treat the problem as a classical stable admissions problem. We model the preferences of the couples indirectly as side-constraints. We look for stable solutions in the college admissions problem where the couples are matched to colleges in the same geographical region.

We use a simple example to motivate our approach. Consider the example discussed in Section 3.2, where the individual preferences for the universities are as shown in Table 1. Suppose further that $\left(a_{4}, a_{10}\right),\left(a_{2}, a_{8}\right)$ are couples. They would like to be assigned to universities in close proximity, so that they can stay together. For the couple $\left(a_{4}, a_{10}\right)$, as far as possible, they would like both to be assigned to universities in the cluster $\left\{u_{3}\right\}$, or to the cluster $\left\{u_{1}, u_{2}, u_{4}, u_{5}\right\}$. Similarly, the couple $\left(a_{2}, a_{8}\right)$ would like both to be assigned to universities in the cluster $\left\{u_{1}, u_{3}\right\}$, or to the cluster $\left\{u_{2}, u_{4}, u_{5}\right\}$.

To incorporate these additional considerations, we need to answer the question: "Is there a stable matching which meets these additional requirements? If so, how do we construct the solution?"

The stable matching $M_{1}$ fails to address the concerns of the couples, since $a_{4}$ is assigned to $u_{3}$, but $a_{10}$ is assigned to $u_{1}$. The matching $M_{7}$ satisfies the additional requirement of couple $\left(a_{4}, a_{10}\right)$ (both are matched to universities in the same cluster $\left\{u_{1}, u_{2}, u_{4}, u_{5}\right\}$ ), but fails to address the needs of couple $\left(a_{2}, a_{8}\right)$. There is only one matching in this problem that meets the additional requirement from both couples: $M_{5}$.

In the rest of this section, we show that under a natural, restricted class of preferences, one can determine efficiently whether or not a stable matching exists by simply solving a related linear program. In particular, as long as the concerns of the couples can be modeled by geographical concerns similar to (a)-(c) defined below, our model can be used to determine whether a feasible solution exists, and if so, construct a feasible solution.

Specifically, we assume that each couple partitions the universities into several groups based on their geographical locations. For example, the couple may choose to group together all of the universities located in the same city. Note that the grouping of the universities can be different for different couples. In our previous example, for instance, the couple $\left(a_{4}, a_{10}\right)$ has chosen to group the universities into clusters $\left\{u_{3}\right\},\left\{u_{1}, u_{2}, u_{4}, u_{5}\right\}$, whereas the couple $\left(a_{2}, a_{8}\right)$ has chosen to group the universities into clusters $\left\{u_{1}, u_{3}\right\}$ and $\left\{u_{2}, u_{4}, u_{5}\right\}$. Each husband and wife submit separate rankings of the universities subject to the following restriction:

(a) The ranking is first done by ranking the group and then ranking the universities in the group;

(b) If a person prefers group 1 to group 2, then all universities deemed acceptable ${ }^{7}$ in group 1 will be ranked above any university deemed acceptable in group 2; and

(c) Each couple must rank the groups in the same way, but are allowed to submit different rankings of the universities within a group.

\footnotetext{
${ }^{7}$ Certain universities in the group may not be acceptable to the student
} 
For each couple $c$, let $\mathcal{K}_{c}=K_{c, 1} \cup K_{c, 2} \cup \ldots \cup K_{c, D}$ denote the groupings of the (acceptable) universities, sorted according to the ranking of the cities. i.e., (acceptable) universities in group $K_{c, i}$ are generally preferred by the couple $c$ to universities in group $K_{c, j}$, if $i<j$. The essential geographic preferences of the couples are captured by allowing the husband and wife to be assigned to different universities as long as these universities are within the same group (i.e., in the same region). This will be modeled as a side constraint in our model.

For ease of exposition, we assume that each couple has partitioned the universities into $D$ groupings. Furthermore, we will assume that the students accept all universities (i.e. complete ranking) they have been assigned to. This condition can be easily relaxed by adding additional constraints of the type $x_{u, a}=0$ if student $a$ does not accept university $u$.

We wish to find a stable matching in which each couple is assigned to universities within the same group. We show that a variation of the linear inequality description for the stable admission polytope remains valid for this version of the stable matching problem with couples; in particular, a stable solution, if it exists, can be found using linear programming.

We claim that the following inequalities give the polytope of stable admission with couples $\left(P_{S A C}\right)$ :

$$
\begin{aligned}
& \sum_{u:(u, a) \in \Gamma} x_{u, a} \leq 1, \quad \forall a \in A, \\
& \sum_{a:(u, a) \in \Gamma} x_{u, a} \leq q_{u}, \quad \forall u \in U, \\
& x(C)=\sum_{(u, a) \in C} x_{u, a} \geq q_{u}, \quad \forall C \in C_{u}, u \in U . \\
& x_{u, a} \geq 0 \quad \forall a,(u, a) \in \Gamma, \\
& \left\{\begin{array}{l}
\sum_{j \in K_{c, l}} x_{p, j}+\sum_{j \notin K_{c, l}} x_{q, j} \leq 1 \\
\sum_{j \in K_{c, l}} x_{q, j}+\sum_{j \notin K_{c, l}} x_{p, j} \leq 1,
\end{array} \quad \text { for all couples }(p, q) \text { and group } K_{c, l} .\right.
\end{aligned}
$$

Here the inequalities $(15,16,17,18,19)$ are called the student, university, comb, non-negativity, and the couple constraints respectively. The first four classes of constraints are basically the valid constraints in the classical many-to-one stable admissions polytope. As long as both students in the couple pair are matched to some universities, then the couple constraints ensure that they are matched to universities in the same geographical locations.

The fractional solution of $\left(P_{S A C}\right)$ still satisfies the decomposition property of section 3.1 , since it is a subset of the stable admission polytope. Note that if a student is unmatched in the stable admission problem (without the added side constraints), then he/she remains unmatched in all the stable matchings. This property ensures that either $\sum_{u} x_{u, a}=0$ or $\sum_{u} x_{u, a}=1$ in $\left(P_{S A C}\right)$.

Consider the randomized rounding method described in Theorem 6: Generate a number $\alpha \in(0,1]$ uniformly at random; for each university $u$, assign the $q_{u}$ students in $I_{u}^{\alpha}(x)$ to university $u$ to get an assignment $\mu_{\alpha}$. Note that by our rounding method will automatically produce a stable matching for the original problem. We need to ensure further that it satisfies the additional couple constraints. 
This is clear whenever $\sum_{u} x_{u, p}=0$ or $\sum_{u} x_{u, q}=0$ for couple $(p, q)$. On the other hand, if $\sum_{u} x_{u, p}=1$ and $\sum_{u} x_{u, q}=1$, then the couple constraints reduce to

$$
\sum_{j \in K_{c, l}} x_{p, j} \leq \sum_{j \in K_{c, l}} x_{q, j}
$$

and

$$
\sum_{j \in K_{c, l}} x_{q, j} \leq \sum_{j \in K_{c, l}} x_{p, j}
$$

i.e.,

$$
\sum_{j \in K_{c, l}} x_{p, j}=\sum_{j \in K_{c, l}} x_{q, j} \text { for all } K_{c, l} .
$$

Since the individual preferences of $p$ and $q$ are obtained by an identical ranking of the groupings first, the above property ensures that in our randomized rounding method, whenever $p$ is assigned to a university in $K_{c, l}, q$ will also be assigned to one in $K_{c, l}$ too.

We thus have the following result.

Theorem 10 The convex hull of the stable admission solutions to the couples problem satisfying conditions (a), (b) and (c) is given by $\left(P_{S A C}\right)$.

When $\left(P_{S A C}\right)=\emptyset$ (when the LP is infeasible), the LP method will return an inconsistent set of inequalities automatically, producing a certificate to the fact that there does not exist any stable solution assigning all couples to the same geographical regions.

The above model for the couples problem inherit all the nice properties for the classical stable admission problem, whenever a feasible solution can be found. In particular, the lattice properties of the feasible stable admission solutions are preserved under this model. Using the same argument in our derivation of the median solution concept, and the fact that $\left(P_{S A C}\right) \subset\left(P_{S A}\right)$, we also have the next result:

Theorem 11 If $\left(P_{S A C}\right) \neq \emptyset$, then a median solution exists for the stable admission problem with couples.

\section{Concluding Remarks}

In this paper, we study the stable admissions problem using a simple geometric property of the fractional solutions in the stable admissions polytope. We exhibit a simple decomposition property, which allows us to express fractional solutions in the stable admissions polytope as convex combinations of integral solutions. The results provide new insight into the stable admissions problem, and generalize many structural properties of the classical stable marriage problem. 
The stable admissions problem can be viewed as a special case of the stable marriage problem with indifference. However, since the problem of determining whether a given pair is stable is NPcomplete for a given instance of stable marriage with ties [27], this appears to be substantially more difficult. It is interesting to find out whether the geometric structure of the fractional solutions carries over to this situation. Another special case of the stable matching problem with indifference is the many-to-many stable matching problem with strict preferences. We believe that the properties of the fractional solutions has an analogous interpretation in this general setting. However, describing the stable matching polytope explicitly in this case requires a non-trivial extension to the comb inequalities. Another interesting open issue is to consider more general preferences for the universities for which the existence of a stable matching is assured; the class of substitutable preferences is a natural candidate. We leave these issues for further research.

\section{Acknowledgements}

We thank Bettina Klaus, Flip Klijn, and the four anonymous referees for their many constructive

suggestions on an earlier version of this paper. We are grateful for their detailed comments, which helped us sharpen and improve the results as well the exposition.

\section{References}

[1] H. Adachi. On a characterization of stable matchings. Economics Letters, 68(1):43-49, 2000.

[2] B. Aldershof and O. M. Carducci. Stable matchings with couples, Discrete Applied Mathematics, 68, 203$207,1996$.

[3] B. Aldershof, O.M. Carducci, and D.C. Lorenc, Refined Inequalities for Stable Marriage, Constraints 4, 281-292, 1999.

[4] A. Abdulkadiroğlu. College Admissions with Affirmative Action. International Journal of Game Theory, 33, 535-549, 2005.

[5] M. Baïou and M. L. Balinski. Many-to-many matching: stable polyandrous polygamy (or polygamous polyandry), Discrete Appl. Math. 101:1-3, 1-12, 2000.

[6] M. Baïou and M. L. Balinski. The stable admissions polytope, Math. Program., Ser. A 87, 427-439, 2000.

[7] M. Balinski and G. Ratier. On stable marriages and graphs, and strategy and polytopes, SIAM Rev. 39(4):575-604, 1997.

[8] M. Balinski and G. Ratier. Graphs and Marriages, American Mathematical Monthly 105(5):430-445, 1998.

[9] G.E. Bolton, J. Brandts and A. Ockenfels. Fair Procedures: Evidence from Games Involving Lotteries. Economic Journal, Vol. 115, 1054-1076, 2005

[10] C. Camerer and R.H. Thaler. Anomalies: Ultimatums, dictators and manners. Journal of Economic Perspectives, 9(2), 209-219, 1995. 
[11] D. Cantala. Matching Markets: the particular case of couples, Economics Bulletin, Vol. 3 no. 45 pp. 1-11, 2004.

[12] T. Feder. A New Fixed Point Approach for Stable Networks and Stable Marriages, Journal of Computer and Systems Sciences, 45(2):233-284, 1992.

[13] T. Feder. Stable Networks and Product Graphs, American Mathematical Society, 1995.

[14] T. Fleiner. A fixed-point approach to stable matchings and some applications, Math. Oper. Res., 28(1):103126, 2003.

[15] T. Fleiner. Some results on stable matchings and fixed points, TR-2002-08, EGRES, December 2002.

[16] T. Fleiner. The stable b-matching polytope, Mathematical Social Science, 46(2):149-158, 2003.

[17] D. Gale and L. S. Shapley. College admissions and the stability of marriage, Amer. Math. Monthly, 69, $9-15,1962$.

[18] D. Gusfield and R. W. Irving. The Stable marriage Problem: Structure and Algorithms, 1989, MIT Press, Massachusetts.

[19] W. Guth, R. Schmittberger and B. Schwarze. An experimental analysis of ultimatum bargaining. Journal of Economic Behavior and Organization 3(4), 36788, 1982.

[20] R. W. Irving, P. Leather and D. Gusfield. An efficient algorithm for the optimal stable marriage problem, J. ACM, 34 (1987) 532-543.

[21] B. Klaus and F. Klijn. Stable Matchings and Preferences of Couples, Journal of Economic Theory, 121(1), 75-106, 2005.

[22] B. Klaus and F. Klijn. Procedurally Fair and Stable Matching, Economic Theory, 27(2), 431-447, 2006.

[23] B. Klaus and F. Klijn. Median Stable Matching for College Admissions Working Paper, Universitat Autonoma de Barcelona, 2004.

[24] B. Klaus, F. Klijn, and J. Masso. Some Things Couples always wanted to know about Stable Matchings (but were afraid to ask), Working Paper, Universitat Autonoma de Barcelona, 2003.

[25] D. E. Knuth and M. Goldstein. Stable Marriage and Its Relation to Other Combinatorial Problems: An Introduction to the Mathematical Analysis of Algorithms, American Mathematical Society, January 1997.

[26] J. Ma. On Randomized Matching Mechanisms, Economic Theory 8 (1996), 377-381.

[27] D. F. Manlove, R. W. Irving, K. Iwama, S. Miyazaki, and Y. Morita. Hard variants of stable marriage, Theoretical Computer science, 276 (2002) 261-279.

[28] A. Romero-Medina. Equitable selection in bilateral matching markets, Forthcoming Theory and Decision.

[29] A. E. Roth. The Evolution of the Labor Market for Medical Interns and Residents: A Case Study in Game Theory, Journal of Political Economy, 92(6):991-1016, 1984.

[30] A. E. Roth. On the Allocation of Residents to Rural hospitals: A General Property of Two-Sided Matching Markets, Econometrica, 54:2 (1986) 425-428. 
[31] A. E. Roth and E. Peranson. The effects of the change in the NRMP matching algorithm, Journal of the American Medical Association, 278(9):729-732, 1997.

[32] A. E. Roth and E. Peranson. The Redesign of the Matching Market for American Physicians: Some Engineering Aspects of Economic Design, American Economic Review, 89(4):748-780, 1999.

[33] A. E. Roth and Sotomayor M. Two-Sided Matching: A Study in game-Theoretic Modeling and Analysis, 1991, Cambridge University Press, Cambridge.

[34] A. E. Roth and Sotomayor M. The college admissions problem revisited, Econometrica, 57, 559-570, 1989.

[35] A. E. Roth, U. G. Rothblum and J. H Vande Vate. Stable matching, optimal assignments and linear programming, Math. Oper. Res., 18 (1993) 808-828.

[36] Roth, A.E. and Vande Vate, J.H. Random Paths to Stability in Two-Sided Matching, Econometrica 58 (1990) 1475-1480.

[37] U. G. Rothblum. Characterization of stable matchings as extreme points of a polytope, Mathematical Programming, 54 (1992), 57-67.

[38] A. Subramanian. A new approach to stable matching problems, SIAM J. Comput., 23 (1994) 671-700.

[39] C.P. Teo and Jay Sethuraman. The geometry of fractional stable matchings and its applications, Math. Oper. Res., 23:4 (1998) 874-891.

[40] J. H. Vande Vate. Linear programming brings marital bliss, Oper. Res. Lett., 8 (1989) 147-153. 